\title{
Exact Solutions of the Symmetric Regularized Long Wave Equation and the Klein-Gordon-Zakharov Equations
}

\author{
Isaiah Elvis Mhlanga and Chaudry Masood Khalique \\ International Institute for Symmetry Analysis and Mathematical Modelling, Department of Mathematical Sciences, \\ North-West University, Mafikeng Campus, Private Bag X 2046, Mmabatho 2735, South Africa
}

Correspondence should be addressed to Chaudry Masood Khalique; masood.khalique@nwu.ac.za

Received 14 February 2014; Accepted 20 March 2014; Published 6 April 2014

Academic Editor: Baojian Hong

Copyright (C) 2014 I. E. Mhlanga and C. M. Khalique. This is an open access article distributed under the Creative Commons Attribution License, which permits unrestricted use, distribution, and reproduction in any medium, provided the original work is properly cited.

We study two nonlinear partial differential equations, namely, the symmetric regularized long wave equation and the Klein-GordonZakharov equations. The Lie symmetry approach along with the simplest equation and exp-function methods are used to obtain solutions of the symmetric regularized long wave equation, while the travelling wave hypothesis approach along with the simplest equation method is utilized to obtain new exact solutions of the Klein-Gordon-Zakharov equations.

\section{Introduction}

The investigation of exact travelling wave solutions of nonlinear partial differential equations (NLPDEs) is important for the understanding of most nonlinear physical phenomena that appear in many areas of scientific fields such as plasma physics, solid state physics, fluid dynamics, optical fibers, mathematical biology, and chemical kinetics $[1,2]$. A number of methods have been developed for finding travelling wave solutions to NLPDEs. These include the homogeneous balance method [3], the ansatz method [4, 5], variable separation approach [6], inverse scattering transform method [2], Bäcklund transformation [7], Darboux transformation [8], Hirota bilinear method [9], the $\left(G^{\prime} / G\right)$-expansion method [10], the reduction $\mathrm{mKdV}$ equation method [11], the trifunction method $[12,13]$, the projective Riccati equation method [14], the sine-cosine method $[15,16]$, the Jacobi elliptic function expansion method $[17,18]$, the $F$-expansion method [19], the exp-function expansion method [20], dynamical system method [21-23], and Lie symmetry method [24-28].

In this paper we study two nonlinear partial differential equations, namely, the symmetric regularized long wave equation and the Klein-Gordon-Zakharov equations. The Lie symmetry approach along with the simplest equation and exp-function methods are used to obtain solutions of the symmetric regularized long wave equation, while the travelling wave hypothesis approach along with the simplest equation method is utilized to obtain new exact solutions of the Klein-Gordon-Zakharov equations.

\section{The Symmetric Regularized Long Wave Equation}

We first consider that the symmetric regularized long wave equation (SRLW) as given by

$$
u_{t t}-u_{x x}+\frac{1}{2}\left(u^{2}\right)_{x t}-u_{x x t t}=0
$$

is a nonlinear evolution equation which arises in several physical applications, for example in sound waves in a plasma [29]. Exact travelling wave solutions of this equation were obtained using the $\left(G^{\prime} / G\right)$-expansion method [29]. In the present work, Lie symmetry method along with the simplest equation method and the exp-function method are used to construct exact solutions for this equation. First the Lie point symmetries of the SRLW equation (1) are found using the Lie algorithm [25]. These Lie point symmetries are then used to transform (1) into an ordinary differential equation. The simplest equation method [30] and the exp-function method [20] are then used to construct exact solutions of the ordinary 
differential equation, which leads to the exact solutions of the SRLW equation.

2.1. Lie Point Symmetries of (1) and Symmetry Reduction. The symmetry group of the SRLW equation (1) is generated by the vector field

$$
X=\xi^{1}(t, x, u) \frac{\partial}{\partial t}+\xi^{2}(t, x, u) \frac{\partial}{\partial x}+\eta(t, x, u) \frac{\partial}{\partial u} .
$$

Applying the fourth prolongation of $X$ to (1) and solving the resultant overdetermined system of linear partial differential equations, we obtain the following two translation symmetries:

$$
\begin{aligned}
& X_{1}=\frac{\partial}{\partial t} \\
& X_{2}=\frac{\partial}{\partial x}
\end{aligned}
$$

Now taking the linear combination of these translation symmetries $X_{1}=\partial / \partial t$ and $X_{2}=\partial / \partial x$, namely, the symmetry $X=X_{1}+v X_{2}$, where $v$ is a constant, leads to the two invariants

$$
z=x-v t, \quad u=F(z) .
$$

Treating $F$ as the new dependent variable and $z$ as the new independent variable and then substituting the value of $u$ into the SRLW equation (1) transform (1) into a fourth-order nonlinear ordinary differential equation:

$$
\left(v^{2}-1\right) F^{\prime \prime}(z)-v F(z) F^{\prime \prime}(z)-v\left(F^{\prime}(z)\right)^{2}+v^{2} F(z)^{\prime \prime \prime \prime}=0
$$

\subsection{Exact Solutions of (1) Using Simplest Equation Method.} Now the simplest equation method $[30,31]$ is used to solve (5), and henceforth one obtains the exact solutions of the SRLW equation (1). The Bernoulli and Riccati equations will be used as the simplest equations. The Bernoulli and Riccati equations are well-known equations whose solutions can be expressed in terms of elementary functions [28].

The Bernoulli equation which we use here is given by

$$
H^{\prime}(z)=c H(z)+d H^{2}(z)
$$

where $c$ and $d$ are constants. Its solution is given by

$$
H(z)=c\left\{\frac{\cosh [c(z+C)]+\sinh [c(z+C)]}{1-d \cosh [c(z+C)]-d \sinh [c(z+C)]}\right\},
$$

where $C$ is a constant of integration [28].

For the Riccati equation

$$
G^{\prime}(z)=c G^{2}(z)+d G(z)+e,
$$

where $c, d$, and $e$ are constants, the solutions to be used are

$$
\begin{gathered}
H(z)=-\frac{d}{2 c}-\frac{\theta}{2 c} \tanh \left[\frac{1}{2} \theta(z+C)\right], \\
H(z)=-\frac{d}{2 c}-\frac{\theta}{2 c} \tanh \left(\frac{1}{2} \theta z\right) \\
+\frac{\operatorname{sech}(\theta z / 2)}{C \cosh (\theta z / 2)-(2 c / \theta) \sinh (\theta z / 2)},
\end{gathered}
$$

with $\theta^{2}=d^{2}-4 c e>0$ and $C$ being a constant of integration [28].

2.2.1. Solutions of (1) Using Bernoulli as the Simplest Equation. The solutions of the ODE (5) are considered to be in the form

$$
F(z)=\sum_{i=0}^{M} \mathscr{A}_{i}(H(z))^{i}
$$

where $H(z)$ satisfies the Bernoulli or Riccati equations, $M$ is a positive integer that can be determined by balancing the highest order derivative term with the highest order nonlinear term [31], and $\mathscr{A}_{i},(i=0,1, \ldots, M)$ are parameters to be determined.

The balancing procedure yields $M=2$, so the solutions of (5) are of the form

$$
F(z)=\mathscr{A}_{0}+\mathscr{A}_{1} H+\mathscr{A}_{2} H^{2}
$$

Substituting (11) into (5), making use of the Bernoulli equation (6), and then equating all coefficients of the function $H^{i}$ to zero, we obtain the following algebraic system of equations in terms of $\mathscr{A}_{0}, \mathscr{A}_{1}$, and $\mathscr{A}_{2}$ :

$$
\begin{aligned}
& -10 v \mathscr{A}_{2}^{2} d^{2}-120 v^{2} \mathscr{A}_{2} d^{4}=0, \\
& -24 v^{2} \mathscr{A}_{1} d^{4}-12 v \mathscr{A}_{1} d^{2} \mathscr{A}_{2} \\
& \quad-336 v^{2} \mathscr{A}_{2} d^{3} c-18 v \mathscr{A}_{2}^{2} d c=0, \\
& -v \mathscr{A}_{0} \mathscr{A}_{1} c^{2}-\mathscr{A}_{1} c^{2}-v^{2} \mathscr{A}_{1} c^{4}+v^{2} \mathscr{A}_{1} c^{2}=0, \\
& -8 v \mathscr{A}_{2}^{2} c^{2}-6 \mathscr{A}_{2} d^{2}+6 v^{2} \mathscr{A}_{2} d^{2}-21 v \mathscr{A}_{1} d \mathscr{A}_{2} c-3 v \mathscr{A}_{1}^{2} d^{2} \\
& \quad-60 v^{2} \mathscr{A}_{1} d^{3} c-6 v \mathscr{A}_{0} \mathscr{A}_{2} d^{2}-330 v^{2} \mathscr{A}_{2} d^{2} c^{2}=0, \\
& -3 \mathscr{A}_{1} d c+3 v^{2} \mathscr{A}_{1} d c-4 v \mathscr{A}_{0} \mathscr{A}_{2} c^{2}-4 \mathscr{A}_{2} c^{2}+4 v^{2} \mathscr{A}_{2} c^{2} \\
& \quad-3 v \mathscr{A}_{0} \mathscr{A}_{1} d c-2 v \mathscr{A}_{1}^{2} c^{2} \\
& -16 v^{2} \mathscr{A}_{2} c^{4}-15 v^{2} \mathscr{A}_{1} d c^{3}=0, \\
& -2 \mathscr{A}_{1} d^{2}-50 v^{2} \mathscr{A}_{1} d^{2} c^{2}+10 v^{2} \mathscr{A}_{2} d c-5 v \mathscr{A}_{1}^{2} d c \\
& -130 v^{2} \mathscr{A}_{2} d c^{3}+2 v^{2} \mathscr{A}_{1} d^{2}-9 v \mathscr{A}_{1} c^{2} \mathscr{A}_{2} \\
& -2 v \mathscr{A}_{0} \mathscr{A}_{1} d^{2}-10 v \mathscr{A}_{0} \mathscr{A}_{2} d c-10 \mathscr{A}_{2} d c=0 .
\end{aligned}
$$


Solving this system, with the aid of Maple, we obtain the following values for the constants:

$$
\begin{aligned}
& \mathscr{A}_{0}=\frac{-c^{2} v^{2}+v^{2}-1}{v}, \\
& \mathscr{A}_{1}=-12 c d v, \\
& \mathscr{A}_{2}=-12 v d^{2} .
\end{aligned}
$$

As a result a solution of the symmetric regularized long wave equation (1) using the Bernoulli equation as the simplest equation is

$$
\begin{aligned}
u(t, x) & \\
= & \mathscr{A}_{0}+\mathscr{A}_{1} c\left\{\frac{\cosh [c(z+C)]+\sinh [c(z+C)]}{1-d \cosh [c(z+C)]-d \sinh [c(z+C)]}\right\} \\
& +\mathscr{A}_{2} c^{2}\left\{\frac{\cosh [c(z+C)]+\sinh [c(z+C)]}{1-d \cosh [c(z+C)]-d \sinh [c(z+C)]}\right\}^{2},
\end{aligned}
$$

where $z=x-v t$ and $C$ is a constant of integration.

2.2.2. Solutions of (1) Using Ricatti as the Simplest Equation. The balancing procedure yields $M=2$, so the solutions of (5) are of the form

$$
F(z)=\mathscr{A}_{0}+\mathscr{A}_{1} G(z)+\mathscr{A}_{2} G^{2}(z)
$$

Substituting (15) into (5), making use of the Ricatti equation (8), and then equating all coefficients of the function $G^{i}$ to zero, we obtain an algebraic system of equations in terms of $\mathscr{A}_{0}, \mathscr{A}_{1}$, and $\mathscr{A}_{2}$. Solving the resultant algebraic equations, we obtain the following set of values:

$$
\begin{aligned}
& \mathscr{A}_{0}=-\frac{8 c e v^{2}+d^{2} v^{2}-v^{2}+1}{v}, \\
& \mathscr{A}_{1}=-12 c d v, \\
& \mathscr{A}_{2}=-12 c^{2} v .
\end{aligned}
$$

It follows that the solutions for the symmetric regularized long wave equation (1) using the Ricatti equation as the simplest equation are

$$
\begin{aligned}
u(t, x)= & \mathscr{A}_{0}+\mathscr{A}_{1}\left\{-\frac{d}{2 c}-\frac{\theta}{2 c} \tanh \left[\frac{1}{2} \theta(z+C)\right]\right\} \\
& +\mathscr{A}_{2}\left\{-\frac{d}{2 c}-\frac{\theta}{2 c} \tanh \left[\frac{1}{2} \theta(z+C)\right]\right\}^{2},
\end{aligned}
$$

$$
\begin{aligned}
u(t, x)=\mathscr{A}_{0}+\mathscr{A}_{1}\{- & \frac{d}{2 c}-\frac{\theta}{2 c} \tanh \left(\frac{1}{2} \theta z\right) \\
& \left.+\frac{\operatorname{sech}(\theta z / 2)}{C \cosh (\theta z / 2)-(2 c / \theta) \sinh (\theta z / 2)}\right\} \\
+ & \mathscr{A}_{2}\left\{-\frac{d}{2 c}-\frac{\theta}{2 c} \tanh \left(\frac{1}{2} \theta z\right)\right. \\
& \left.+\frac{\operatorname{sech}(\theta z / 2)}{C \cosh (\theta z / 2)-(2 c / \theta) \sinh (\theta z / 2)}\right\}^{2},
\end{aligned}
$$

where $z=x-v t$ with $\theta^{2}=d^{2}-4 c e>0$ and $C$ is a constant of integration.

2.3. Solution of (1) Using the Exp-Function Method. In this section we use the exp-function method [20] to solve the symmetric regularized long wave equation (1). We consider solutions of (5) in the form

$$
F(z)=\frac{\sum_{n=-b}^{c} a_{n} e^{n z}}{\sum_{m=-p}^{q} b_{m} e^{m z}},
$$

where $b, c, p$, and $q$ are positive integers to be determined and $a_{n}$ and $b_{m}$ are arbitrary constants [20]. The balancing procedure of the exp-function method produces $p=b$ and $q=c$. For simplicity, we set $p=b=1$ and $q=c=1$ so that (18) is reduced to

$$
F(z)=\frac{a_{1} e^{z}+a_{0}+a_{-1} e^{-z}}{b_{1} e^{z}+b_{0}+b_{-1} e^{-z}} .
$$

Substituting (19) into (5) and solving the resultant ODE, with the help of Maple, one possible set of values of the constants is

$$
\begin{aligned}
& a_{-1}=-\frac{b_{-1}}{v}, \\
& a_{0}=\frac{b_{0}\left(6 v^{2}-1\right)}{v}, \\
& a_{1}=\frac{-b_{0}^{2}}{4 v b_{-1}} \\
& b_{1}=\frac{b_{0}^{2}}{4 b_{-1}} .
\end{aligned}
$$

As a result we obtain the solution

$$
u(t, x)=\frac{a_{1} e^{x-\nu t}+a_{0}+a_{-1} e^{-(x-v t)}}{b_{1} e^{x-\nu t}+b_{0}+b_{-1} e^{-(x-\nu t)}} .
$$

\section{The Klein-Gordon-Zakharov Equations}

The Klein-Gordon-Zakharov (KGZ) equations [32]

$$
\begin{gathered}
u_{t t}-u_{x x}+u+u v+|u|^{2} u=0, \\
v_{t t}-v_{x x}-\left(|u|^{2}\right)_{x x}=0,
\end{gathered}
$$


are a coupled system of nonlinear partial differential equations of two functions $u(x, t)$ and $v(x, t)$. This model describes the interaction of the Langmuir wave and the ion acoustic wave in plasma. The function $u(x, t)$ denotes the fast time scale component of electric field raised by electrons and the function $v(x, t)$ denotes the deviation of ion density from its equilibrium. Here $u(x, t)$ is a complex function and $v(x, t)$ is a real function. Note that if we remove the term $|u|^{2} u$, then this system reduces to the classical Klein-Gordon-Zakharov system [33]

$$
\begin{gathered}
u_{t t}-u_{x x}+u+u v=0, \\
v_{t t}-v_{x x}-\left(|u|^{2}\right)_{x x}=0 .
\end{gathered}
$$

A number of studies have been conducted for this system ((23a) and (23b)) in different time space [34-38]. However, for the KGZ equations (22a) and (22b), Chen [39] considered orbital stability of solitary waves, while Shi et al. [33] employed the sine-cosine method and the extended tanh method to construct exact solutions of the KGZ equations (22a) and (22b).

In this paper, we employ an entirely different approach, namely, the travelling wave variable approach along with the simplest equation method to obtain exact solutions of the KGZ equations (22a) and (22b).

3.1. Solution of (22a) and (22b) Using the Travelling Wave Variable Approach. The travelling wave variable approach converts the system of nonlinear partial differential equations into a system of nonlinear ordinary differential equations, which we then solve to obtain exact solutions of the system.

In order to solve the KGZ equations (22a) and (22b), we first transform it into a system of nonlinear ordinary differential equations which can then be solved in order to obtain its exact solutions.

We make the wave variable transformation

$$
\begin{aligned}
u & =e^{i \phi} u(z), & v & =v(z), \\
\phi & =p x+r t, & z & =k x+d t,
\end{aligned}
$$

where $p, r, k$, and $d$ are real constants and $d \neq k$. Using this transformation, (22a) and (22b) transform into

$$
\begin{gathered}
\left(p^{2}-r^{2}+1\right) u+i(2 r d-2 p k) u^{\prime} \\
+\left(d^{2}-k^{2}\right) u^{\prime \prime}+u v+u^{3}=0 \\
\left(d^{2}-k^{2}\right) v^{\prime \prime}-\left(u^{2}\right)^{\prime \prime}=0 .
\end{gathered}
$$

Integrating (25b) twice and taking the constants of integration to be zero, we obtain

$$
v=\frac{u^{2}}{d^{2}-k^{2}} .
$$

Now substituting (26) into (25a), we get

$$
u^{\prime \prime}=\left(\frac{r^{2}-p^{2}-1}{d^{2}-k^{2}}\right) u+\left(\frac{d^{2}-k^{2}+1}{\left(d^{2}-k^{2}\right)^{2}}\right) u^{3},
$$

which can be written in the form

$$
u^{\prime \prime}=P u+Q u^{3}
$$

where

$$
P=\frac{r^{2}-p^{2}-1}{d^{2}-k^{2}}, \quad Q=\frac{d^{2}-k^{2}+1}{\left(d^{2}-k^{2}\right)^{2}} .
$$

Solving (28), with the aid of Mathematica, we obtain the solution

$$
u(z)= \pm \frac{1}{H} i \operatorname{sn}(F \mid \omega)
$$

where $\operatorname{sn}(F \mid \omega)$ is a Jacobian elliptic function of the sine amplitude [40],

$$
\begin{aligned}
& F=\frac{\sqrt{\left(\sqrt{P^{2}-2 Q c_{1}}-P\right)\left(z+c_{2}\right)^{2}}}{\sqrt{2}}, \\
& H=\sqrt{\frac{Q}{\sqrt{P^{2}-2 Q c_{1}}+P}}, \\
& \omega=\frac{-Q c_{1}+P\left(\sqrt{P^{2}-2 Q c_{1}}+P\right)}{Q c_{1}}
\end{aligned}
$$

is the modulus of the elliptic function with $0<\omega<1$. Here $c_{1}$ and $c_{2}$ are constants of integration. Reverting back to our original variables, we can now write the solution of our KleinGordon-Zakharov equations as

$$
u(x, t)= \pm \frac{1}{H} i \operatorname{sn}(F \mid \omega)
$$

where

$$
F=\frac{\sqrt{\left(\sqrt{P^{2}-2 Q c_{1}}-P\right)\left(k x+d t+c_{2}\right)^{2}}}{\sqrt{2}},
$$

and $\omega$ and $H$ are as above.

Now $v(x, t)$ can be obtained from (26).

It should be noted that the solution (32) is valid for $0<$ $\omega<1$, as $\omega$ approaches zero, the solution becomes the normal sine function, $\sin z$, and as $\omega$ approaches 1 , the solution tends to the $\tanh$ function, $\tanh z$.

The profile of the solution (32) is given in Figure 1.

3.2. Solutions of (22a) and (22b) Using the Simplest Equation Method. We consider the solutions of (27) in the form

$$
u(z)=\sum_{i=0}^{M} A_{i}(G(z))^{i}
$$

where $G(z)$ satisfies the Bernoulli or the Riccati equation. 


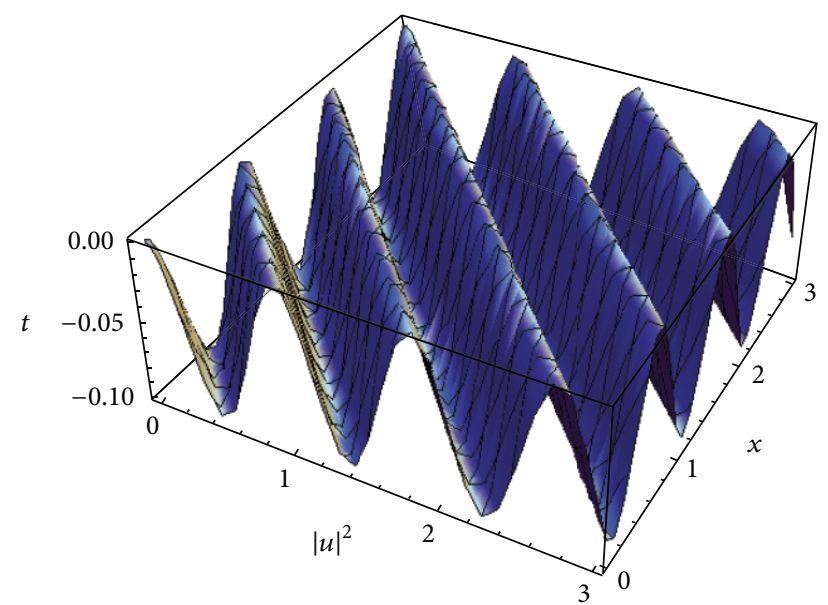

Figure 1: Profile of solution (32).

3.2.1. Solutions of (22a) and (22b) Using Bernoulli as the Simplest Equation. We consider the Bernoulli equation

$$
G^{\prime}(z)=a G(z)+b G^{2}(z)
$$

where $a$ and $b$ are constants.

The balancing procedure yields $M=1$, so the solution of (27) is of the form

$$
u(z)=A_{0}+A_{1} H(z)
$$

Substituting (36) into (27), making use of the Bernoulli equation (35), and then equating all coefficients of the function $H^{i}$ to zero, we obtain the following algebraic system of equations:

$$
\begin{aligned}
& 2 A_{1} k^{4} b^{2}-k^{2} A_{1}^{3}+d^{2} A_{1}^{3}-4 A_{1} d^{2} k^{2} b^{2} \\
& \quad+A_{1}^{3}+2 A_{1} d^{4} b^{2}=0, \\
& 3 A_{1} k^{4} a b+3 d^{2} A_{0} A_{1}^{2}-3 k^{2} A_{0} A_{1}^{2}-6 A_{1} d^{2} k^{2} a b \\
& \quad+3 A_{0} A_{1}^{2}+3 A_{1} d^{4} a b=0, \\
& p^{2} A_{0} d^{2}-p^{2} A_{0} k^{2}-r^{2} A_{0} d^{2}+r^{2} A_{0} k^{2}-A_{0} k^{2} \\
& \quad+d^{2} A_{0}^{3}-k^{2} A_{0}^{3}+A_{0} d^{2}+A_{0}^{3}=0, \\
& A_{1} d^{2}+3 d^{2} A_{0}^{2} A_{1}-p^{2} A_{1} k^{2}+r^{2} A_{1} k^{2}+p^{2} A_{1} d^{2} \\
& +A_{1} d^{4} a^{2}-3 k^{2} A_{0}^{2} A_{1}+A_{1} k^{4} a^{2}+3 A_{0}^{2} A_{1} \\
& -2 A_{1} d^{2} k^{2} a^{2}-r^{2} A_{1} d^{2}-A_{1} k^{2}=0 .
\end{aligned}
$$

Solving this system, with the aid of Maple, we obtain the following values for the constants:

$$
\begin{aligned}
A_{0} & =\frac{a\left(d^{2}-k^{2}\right)}{\sqrt{2\left(k^{2}-d^{2}-1\right)}}, \\
A_{1} & =\frac{\sqrt{2} b\left(d^{2}-k^{2}\right)}{k^{2}-d^{2}-1}, \\
p & =\sqrt{\frac{r^{2} d^{2}-r^{2} k^{2}+k^{2}-d^{2} A_{0}^{2}+k^{2} A_{0}^{2}-d^{2}-A_{0}^{2}}{d^{2}-k^{2}}} .
\end{aligned}
$$

As a result, a solution of the Klein-Gordon-Zakharov equations (22a) and (22b), using the Bernoulli equation as the simplest equation, is

$$
\begin{gathered}
u(x, t) \\
=e^{i(p x+r t)}\left[\left(\left(\sqrt{2} a b\left(d^{2}-k^{2}\right)(\cosh (a(k x+d t+c))\right.\right.\right. \\
+\sinh (a(k x+d t+c)))) \\
\times\left(\sqrt{k^{2}-d^{2}-1}(1-b \cosh (a(k x+d t+c))\right. \\
\left.+\frac{a\left(d^{2}-k^{2}\right)}{\sqrt{2\left(k^{2}-d^{2}-1\right)}}\right],
\end{gathered}
$$

where $c$ is a constant of integration.

3.2.2. Solutions of (22a) and (22b) Using Riccati as the Simplest Equation. We use the Riccati equation given by

$$
G^{\prime}(z)=a G^{2}(z)+b G(z)+c,
$$

where $a, b$, and $c$ are constants. The balancing procedure yields $M=1$, so the solution of (27) is of the form

$$
u(z)=A_{0}+A_{1} G(z) .
$$

Similar calculations yield the following set of values:

$$
\begin{aligned}
A_{0} & =\frac{b\left(d^{2}-k^{2}\right)}{\sqrt{2\left(k^{2}-d^{2}-1\right)}}, \\
A_{1} & =-\frac{\sqrt{2} a\left(d^{2}-k^{2}\right) \sqrt{k^{2}-d^{2}-1}}{d^{2}-k^{2}+1} \\
c & =-\frac{\sqrt{2\left(k^{2}-d^{2}-1\right)}\left(d^{2} b^{2}-k^{2} b^{2}-2-2 p^{2}+2 r^{2}\right)}{4 A_{1}\left(d^{2}-k^{2}+1\right)} .
\end{aligned}
$$


As a result the two solutions of (22a) and (22b) are

$$
\begin{aligned}
& u(x, t)=e^{i \phi}\left[A_{0}+A_{1}\left\{-\frac{b}{2 a}-\frac{\theta}{2 a} \tanh \left[\frac{1}{2} \theta(z+C)\right]\right\}\right], \\
& u(x, t) \\
& =e^{i \phi}\left[A_{0}\right. \\
& +A_{1}\left\{-\frac{b}{2 a}-\frac{\theta}{2 a} \tanh \left(\frac{1}{2} \theta z\right)\right. \\
& \left.\left.\quad+\frac{\operatorname{sech}(\theta z / 2)}{C \cosh (\theta z / 2)-(2 a / \theta) \sinh (\theta z / 2)}\right\}\right],
\end{aligned}
$$

where $\phi=p x+r t$ and $z=k x+d t . \theta$ is given by $\sqrt{b^{2}-4 a c}$, $C$ is a constant of integration, and $A_{0}$ and $A_{1}$ are as obtained above.

It should be noted that by substituting the above value of $u(x, t)$ into (26), one can now obtain the solution for the variable $v(x, t)$.

\section{Conclusion}

In this paper we studied two nonlinear partial differential equations. Firstly, Lie symmetry approach along with the simplest equation and the Exp-function method were used to obtain travelling wave solutions of the symmetric regularized long wave equation. Secondly, the travelling wave hypothesis approach along with the simplest equation method is utilized to obtain new exact solutions of the Klein-Gordon-Zakharov equations.

\section{Conflict of Interests}

The authors declare that there is no conflict of interests regarding the publication of this paper.

\section{Acknowledgments}

Isaiah Elvis Mhlanga would like to thank the Faculty Research Committee, FAST, North-West University, Mafikeng Campus, for the financial support. Chaudry Masood Khalique thanks the North-West University, Mafikeng Campus, for its continued support.

\section{References}

[1] L. Debnath, Nonlinear Partial Differential Equations for Scientists and Engineers, Birkhäuser, Boston, Mass, USA, 1997.

[2] M. J. Ablowitz and P. A. Clarkson, Solitons, Nonlinear Evolution Equations and Inverse Scattering, vol. 149 of London Mathematical Society Lecture Note Series, Cambridge University Press, Cambridge, UK, 1991.

[3] M. Wang, Y. Zhou, and Z. Li, "Application of a homogeneous balance method to exact solutions of nonlinear equations in mathematical physics," Physics Letters A: General, Atomic and Solid State Physics, vol. 216, no. 1-5, pp. 67-75, 1996.
[4] J. Hu, "Explicit solutions to three nonlinear physical models," Physics Letters A, vol. 287, no. 1-2, pp. 81-89, 2001.

[5] J. Hu and H. Zhang, "A new method for finding exact traveling wave solutions to nonlinear partial differential equations," Physics Letters A, vol. 286, no. 2-3, pp. 175-179, 2001.

[6] S.-Y. Lou and J. Lu, "Special solutions from the variable separation approach: the Davey-Stewartson equation," Journal of Physics A: Mathematical and General, vol. 29, no. 14, pp. 42094215, 1996.

[7] C. H. Gu, H. S. Hu, and Z. X. Zhou, Soliton Theory and Its Application, Zhejiang Science and Technology Press, Zhejiang, China, 1990.

[8] V. B. Matveev and M. A. Salle, Darboux Transformations and Solitons, Springer Series in Nonlinear Dynamics, Springer, Berlin, Germany, 1991.

[9] R. Hirota, The Direct Method in Soliton Theory, vol. 155 of Cambridge Tracts in Mathematics, Cambridge University Press, Cambridge, UK, 2004.

[10] E. M. E. Zayed and K. A. Gepreel, "The $\left(G^{\prime} / G\right)$-expansion method for finding traveling wave solutions of nonlinear partial differential equations in mathematical physics," Journal of Mathematical Physics, vol. 50, Article ID 013502, 2009.

[11] Z. Yan, "A reduction mKdV method with symbolic computation to construct new doubly-periodic solutions for nonlinear wave equations," International Journal of Modern Physics C, vol. 14, no. 5, pp. 661-672, 2003.

[12] Z. Yan, "The new tri-function method to multiple exact solutions of nonlinear wave equations," Physica Scripta, vol. 78, Article ID 035001, 2008.

[13] Z. Yan, "Periodic, solitary and rational wave solutions of the 3D extended quantum Zakharov-Kuznetsov equation in dense quantum plasmas," Physics Letters A: General, Atomic and Solid State Physics, vol. 373, no. 29, pp. 2432-2437, 2009.

[14] D. Lu and B. Hong, "New exact solutions for the $(2+1)$ dimensional generalized Broer-Kaup system," Applied Mathematics and Computation, vol. 199, no. 2, pp. 572-580, 2008.

[15] A.-M. Wazwaz, "The tanh and the sine-cosine methods for compact and noncompact solutions of the nonlinear KleinGordon equation," Applied Mathematics and Computation, vol. 167, no. 2, pp. 1179-1195, 2005.

[16] Z. Yan and H. Zhang, "New explicit solitary wave solutions and periodic wave solutions for Whitham-Broer-Kaup equation in shallow water," Physics Letters A, vol. 285, no. 5-6, pp. 355-362, 2001.

[17] D. Lü, "Jacobi elliptic function solutions for two variant Boussinesq equations," Chaos, Solitons and Fractals, vol. 24, no. 5, pp. 1373-1385, 2005.

[18] Z. Yan, "Abundant families of Jacobi elliptic function solutions of the $(2+1)$-dimensional integrable Davey-Stewartson-type equation via a new method," Chaos, Solitons and Fractals, vol. 18, no. 2, pp. 299-309, 2003.

[19] M. Wang and X. Li, "Extended F-expansion method and periodic wave solutions for the generalized Zakharov equations," Physics Letters A, vol. 343, no. 1-3, pp. 48-54, 2005.

[20] J.-H. He and X.-H. Wu, "Exp-function method for nonlinear wave equations," Chaos, Solitons \& Fractals, vol. 30, no. 3, pp. 700-708, 2006.

[21] J. Li and H. Dai, On the Study of Singular Nonlinear Traveling Wave Equations. Dynamical System Approach, Science Press, Beijing, China, 2007. 
[22] L. Zhang and L.-Q. Chen, "Envelope compacton and solitary pattern solutions of a generalized nonlinear Schrödinger equation," Nonlinear Analysis: Theory, Methods \& Applications, vol. 70, no. 1, pp. 492-496, 2009.

[23] L. Zhang, L.-Q. Chen, and X. Huo, "The effects of horizontal singular straight line in a generalized nonlinear Klein-Gordon model equation," Nonlinear Dynamics, vol. 72, no. 4, pp. 789801, 2013.

[24] G. W. Bluman and S. Kumei, Symmetries and Differential Equations, vol. 81 of Applied Mathematical Sciences, Springer, New York, NY, USA, 1989.

[25] P. J. Olver, Applications of Lie Groups to Differential Equations, vol. 107 of Graduate Texts in Mathematics, Springer, New York, NY, USA, 2nd edition, 1993.

[26] N. H. Ibragimov, CRC Handbook of Lie Group Analysis of Differential Equations, vol. 1-3, CRC Press, Boca Raton, Fla, USA, 1994-1996.

[27] L. V. Ovsiannikov, Group Analysis of Differential Equations, Academic Press, New York, NY, USA, 1982.

[28] A. R. Adem and C. M. Khalique, "Symmetry reductions, exact solutions and conservation laws of a new coupled KdV system," Communications in Nonlinear Science and Numerical Simulation, vol. 17, no. 9, pp. 3465-3475, 2012.

[29] F. Chand and A. K. Malik, "Exact traveling wave solutions of some nonlinear equations using $\left(G^{\prime} / G\right)$-expansion method methods," International Journal of Nonlinear Science, vol. 14, no. 4, pp. 416-424, 2012.

[30] N. A. Kudryashov, "Simplest equation method to look for exact solutions of nonlinear differential equations," Chaos, Solitons and Fractals, vol. 24, no. 5, pp. 1217-1231, 2005.

[31] N. K. Vitanov and Z. I. Dimitrova, "Application of the method of simplest equation for obtaining exact traveling-wave solutions for two classes of model PDEs from ecology and population dynamics," Communications in Nonlinear Science and Numerical Simulation, vol. 15, no. 10, pp. 2836-2845, 2010.

[32] T. Wang, J. Chen, and L. Zhang, "Conservative difference methods for the Klein-Gordon-Zakharov equations," Journal of Computational and Applied Mathematics, vol. 205, no. 1, pp. 430-452, 2007.

[33] Q. Shi, Q. Xiao, and X. Liu, "Extended wave solutions for a nonlinear Klein-Gordon-Zakharov system," Applied Mathematics and Computation, vol. 218, no. 19, pp. 9922-9929, 2012.

[34] G. Boling and Y. Guangwei, "Global smooth solution for the Klein-Gordon-Zakharov equations," Journal of Mathematical Physics, vol. 36, no. 8, pp. 4119-4124, 1995.

[35] T. Ozawa, K. Tsutaya, and Y. Tsutsumi, "Normal form and global solutions for the Klein-Gordon-Zakharov equations," Annales de l'Institut Henri Poincaré: Analyse Non Linéaire, vol. 12, no. 4, pp. 459-503, 1995.

[36] K. Tsutaya, "Global existence of small amplitude solutions for the Klein-Gordon-Zakharov equations," Nonlinear Analysi: Theory, Methods \& Applications, vol. 27, no. 12, pp. 1373-1380, 1996.

[37] G. Adomian, "Non-perturbative solution of the Klein-GordonZakharov equation," Applied Mathematics and Computation, vol. 81, no. 1, pp. 89-92, 1997.

[38] Y. Shang, Y. Huang, and W. Yuan, "New exact traveling wave solutions for the Klein-Gordon-Zakharov equations," Computers \& Mathematics with Applications, vol. 56, no. 5, pp. 14411450, 2008.
[39] L. Chen, "Orbital stability of solitary waves for the KleinGordon-Zakharov equations," Acta Mathematicae Applicatae Sinica, vol. 15, no. 1, pp. 54-64, 1999.

[40] I. S. Gradshteyn and I. M. Ryzhik, Table of Integrals, Series, and Products, Academic Press, New York, NY, USA, 17th edition, 2007. 


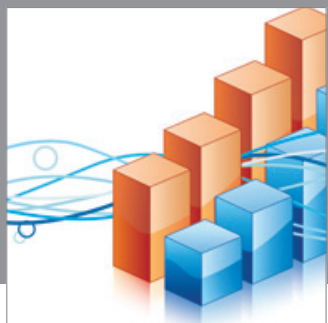

Advances in

Operations Research

mansans

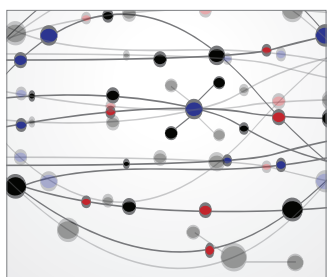

The Scientific World Journal
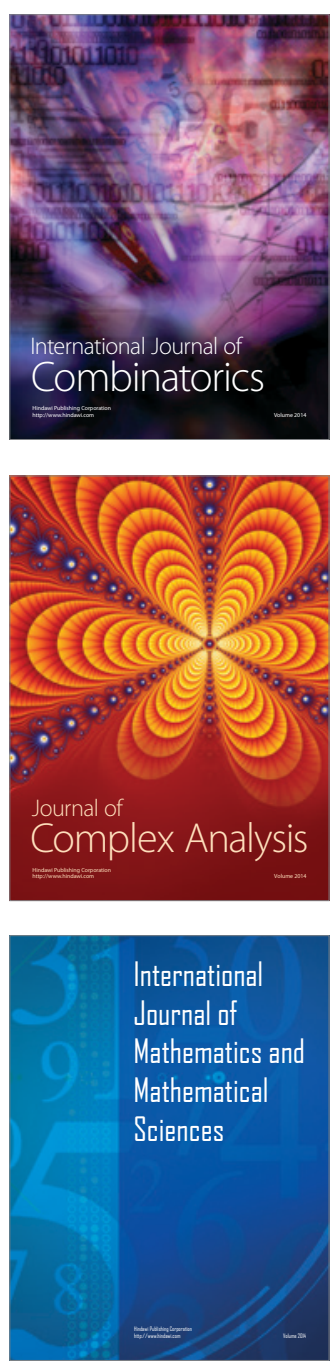
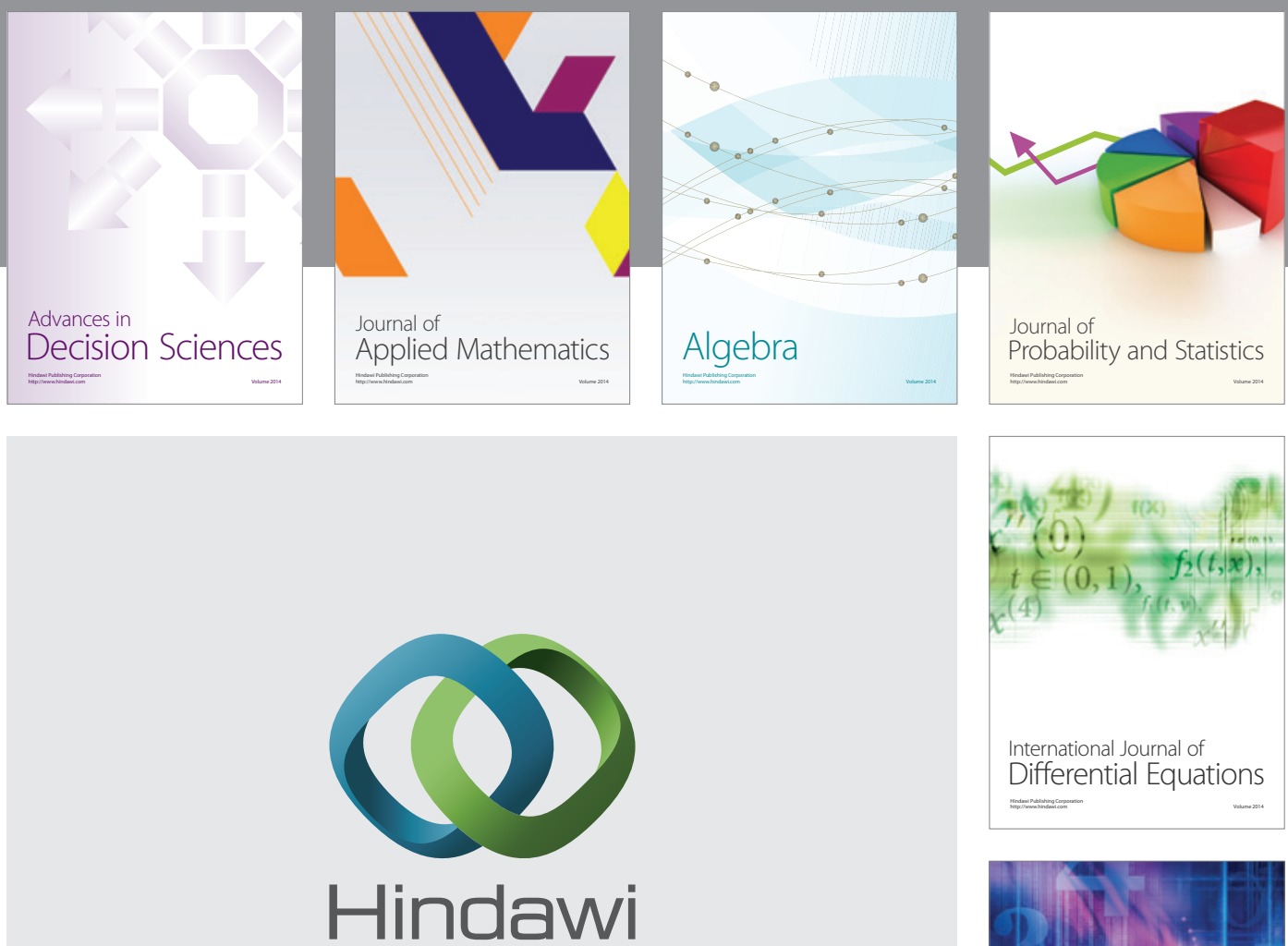

Submit your manuscripts at http://www.hindawi.com
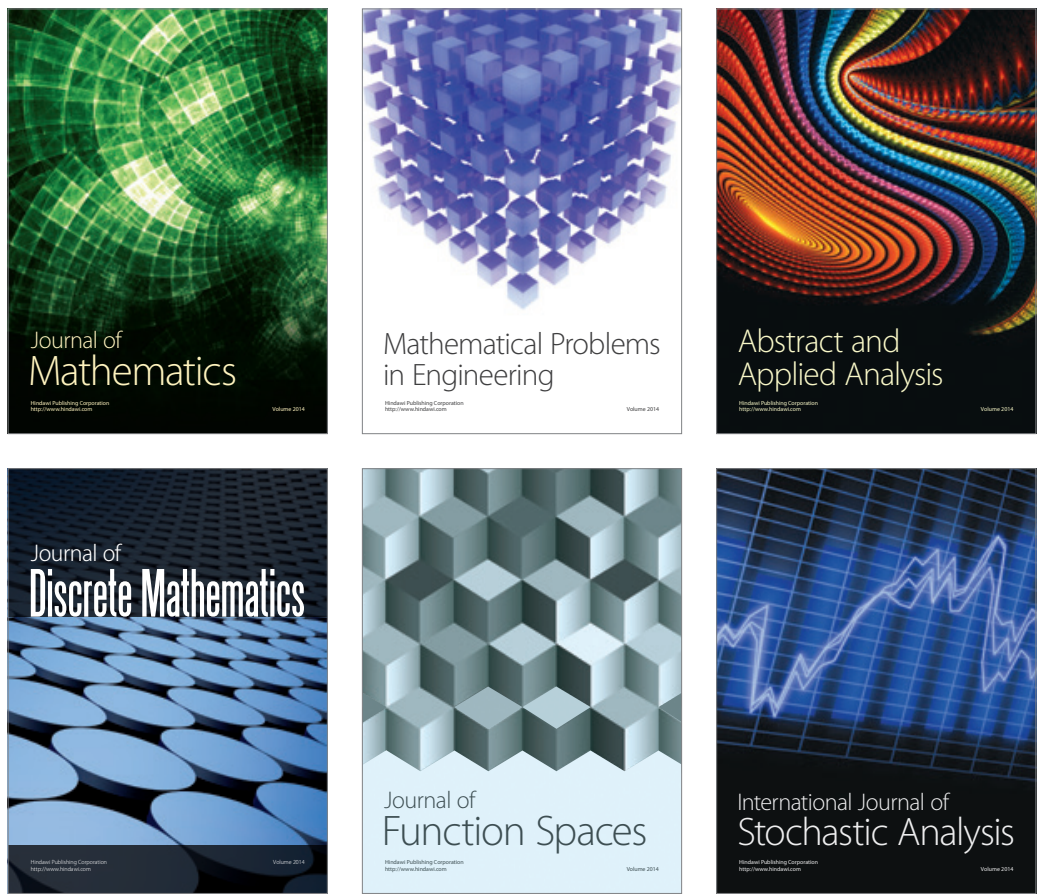

Journal of

Function Spaces

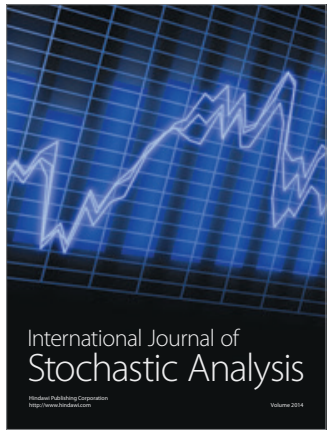

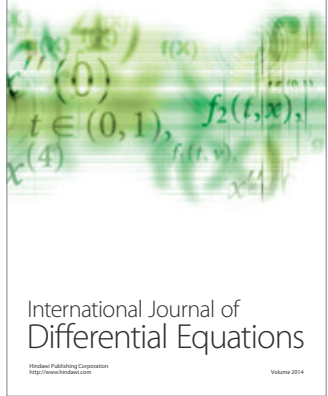
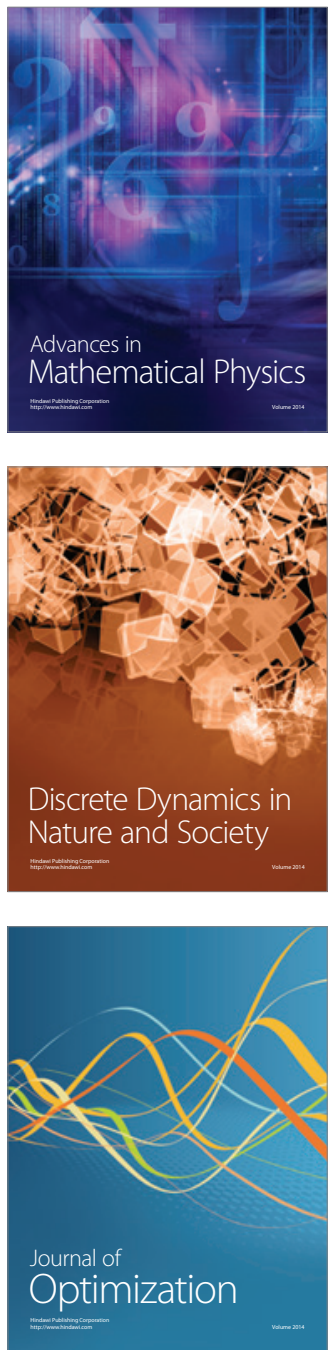